\title{
Propiedades psicométricas del PERMA-Profiler para la medición del bienestar en una muestra de estudiantes universitarios chilenos
}

\author{
Psychometric Properties of the PERMA-Profiler \\ for the Measurement of Wellbeing in a Sample \\ of Chilean University Students
}

Propriedades psicométricas do PERMA-Profiler para a medição do bem-estar em uma amostra de estudantes universitários chilenos

\author{
Rubia Cobo-Rendón, MSc* \\ María Victoria Pérez Villalobos, PhD $^{2}$ \\ Alejandro Díaz Mujica, PhD²
}

Recibido: 18 de julio de 2019 - Aceptado: 10 de octubre de 2019

Doi: https://doi.org/10.12804/revistas.urosario.edu.co/revsalud/a.8775

Para citar este artículo: Cobo-Rendón R, Pérez Villalobos MV, Díaz Mujica A. Propiedades psicométricas del perma-Profiler para la medición del bienestar en una muestra de estudiantes universitarios chilenos. Rev Cienc Salud. 2020; 18(1): 119-133. https://doi. org/10.12804/revistas.urosario.edu.co/revsalud/a.8775

\section{Resumen}

Introducción: en términos científicos y sociales, la investigación del bienestar ha sido relevante en los últimos años, lo que hace necesario investigar sobre las propiedades psicométricas de los cuestionarios que se utilizan para su medición. El objetivo de este trabajo fue estimar las propiedades psicométricas del cuestionario PERMA-Profiler en una muestra de estudiantes universitarios chilenos. Materiales y métodos: se evaluaron las respuestas de 1462 estudiantes universitarios de una universidad tradicional ubicada al sur de Chile, con una edad media de 19.09 años (DE=1.72). Se realizó un análisis factorial confirmatorio, se evaluaron las correlaciones dimensión/total del cuestionario e índices de consistencia interna. Resultados: el análisis factorial reporta la presencia de cinco factores. Se presentaron niveles de correlación moderada, positiva y significativa entre las dimensiones del PERMA-Profiler. Los índices de consistencia de la escala total y de la mayoría de las dimensiones se ubicaron entre $\alpha=.65$ a .91 y de $\omega=.66$ a .92, a excepción de la dimensión de compromiso. Conclusión:

1 Facultad de Ciencias Sociales, Programa de Doctorado en Psicología, Universidad de Concepción, Chile. Autor de correspondencia: rubiacobo@udec.cl; rubiacobo@gmail.com

2 Facultad de Ciencias Sociales, Departamento de Psicología, Universidad de Concepción, Chile. 
se confirman los cinco factores propuestos en la teoría y un adecuado nivel de correlación entre sus dimensiones y de fiabilidad del cuestionario. El PERMA-Profiler puede ser empleado para la medición de la salud mental de los estudiantes y como una herramienta válida para la evaluación de intervenciones en este contexto.

Palabras clave: bienestar; estudiantes; validación; salud del estudiante; promoción de salud.

\section{Abstract}

Introduction: In scientific and social terms, well-being research has been relevant in recent years, which makes it necessary to investigate the psychometric properties of the questionnaires used for its measurement. The objective of this work was to estimate the psychometric properties of the PERMA-Profiler questionnaire in a sample of Chilean university students. Materials and methods: The responses of 1462 university students from a traditional university located in southern Chile were evaluated, with an average age of 19.09 years ( $S D=1.72$ ). A confirmatory factor analysis was performed, and the dimension/ total correlations of the questionnaire and internal consistency indices were evaluated. Results: The factor analysis reports the presence of five factors. There were levels of moderate, positive, and significant correlation between the dimensions of the PERMA-Profiler. The consistency indices of the total scale and most of the dimensions were between $\alpha=.65$ to .91 and from $\omega=.66$ to .92 , with the exception of the commitment dimension. Conclusion: The five factors proposed in theory were confirmed: an adequate level of correlation between its dimensions and the questionnaire's reliability. The PERMA-Profiler can be used to measure students' mental health and as a valid tool for evaluating interventions in this context.

Keywords: Wellbeing; students; validation; student health; health promotion.

\section{Resumo}

Introdução: em termos científicos e sociais a pesquisa do bem-estar tem disso relevante nos últimos anos, o que faz necessário a pesquisa sobre as propriedades psicométricas dos questionários que se utilizam para sua medição. $\mathrm{O}$ objetivo deste trabalho foi estimar as propriedades psicométricas do questionário PERMA-Profiler em uma amostra de estudantes universitários chilenos. Materiais e métodos: avaliaram-se as respostas de 1462 estudantes universitários de uma universidade tradicional localizada no sul do Chile, com idade média de 19.09 anos ( $\mathrm{DE}=1.72$ ). Realizou-se uma análise fatorial confirmatória, avaliaram-se as correlações dimensão/total do questionário e índices de consistência interna. Resultados: a análise fatorial reporta a presença de cinco fatores. Se apresentaram níveis de correlação moderada, positiva e significativa entre as dimensões do PERMA-Profiler. Os índices de consistência da escala total e da maioria das dimensões localizaram-se entre $\alpha=.65$ a .91 y de $\omega=.66$ a .92 , com exceção da dimensão de compromisso. Conclusão: confirmam-se os cinco fatores propostos na teoria, um adequado nível de correlação entre suas dimensões e de fiabilidade do questionário. O PERMA-Profiler pode ser empregado para a medição de saúde mental dos estudantes e como uma ferramenta válida para a avaliação de intervenções neste contexto.

Palavras-chave: bem-estar; estudantes; validação; saúde do estudante; promoção de saúde.

\section{Introducción} I lingreso a la universidad está asociado con mayores niveles de ansiedad, estrés y deprela salud mental, el bienestar se ha presentado como una variable relevante. Estudios recientes reportan que un alto bienestar se asocia a mayores niveles de satisfacción con la vida, afrontamiento al estrés centrado en el problema y menor riesgo de padecer trastornos del 
estado de ánimo (3-6). En términos académicos, el bienestar se asocia con la autorregulación del aprendizaje, la adaptación a la universidad, el éxito estudiantil y la permanencia universitaria (7-13).

La investigación del bienestar ha tenido dos enfoques principales: el "bienestar subjetivo”, fundamentado en la presencia de emociones positivas y satisfacción con la vida, y el "bienestar psicológico" centrado en el desarrollo del potencial humano $(14,15)$. Investigar este tema desde un solo enfoque genera limitaciones en la comprensión del concepto, ya que no puede ser englobado en un aspecto $(16,17)$.

La investigación del bienestar bajo un enfoque multidimensional permite el desarrollo de teorías integradoras (18). En la práctica, se ha dado un mayor reconocimiento al bienestar en la formulación de políticas públicas sobre aspectos que afectan la vida de las personas (19). A pesar de estos avances, todavía existe escasa evidencia psicométrica de estas escalas (20). El presente estudio se justifica por la relevancia que tiene validar las medidas del bienestar en estudiantes universitarios chilenos, teniendo claro que el bienestar es un constructo relevante en la investigación de la salud mental y en el desarrollo de intervenciones para su promoción en este contexto. El desarrollo de estudios psicométricos sobre escalas que miden el bienestar beneficia la comprensión de este constructo y su papel en los distintos niveles de formación educativa (21-22).

Una de las conceptualizaciones propuestas para el estudio del bienestar multidimensional es el modelo PERMA, acrónimo en inglés de emociones positivas, compromiso, relaciones positivas, propósito y logro, definido como la búsqueda y alcance de uno o más de sus cinco elementos (23, 24). Desde su descripción en el 2011, el modelo PERMA aumentó su presencia en el discurso psicológico, pero todavía no existe suficiente apoyo empírico del modelo (25). En los últimos años se han presentado estudios que miden este enfoque con ítems de escalas validadas de dimensiones específicas (26-28).

El PERMA-profiler es un cuestionario que evalúa las dimensiones del PERMA además de una medición global del constructo del bienestar (18). Los autores de esta escala señalan resultados favorables en términos psicométricos, pues tiene índices adecuados de consistencia interna, así como una estructura factorial similar a la propuesta teórica (21). Adicionalmente, se han publicado otros estudios de validación del PERMA-Profiler; por ejemplo, en Estados Unidos se realizó una validación en una muestra de estudiantes veteranos, en la cual los autores reportaron adecuados índices de fiabilidad y validez del instrumento (29). En Malasia, 342 participantes, de los cuales el 50 \% eran estudiantes, completaron el PERMA-Profiler. El análisis factorial exploratorio con el método de componentes principales y rotación oblimin reportó una estructura de tres factores (emociones / relaciones positivas, significado / logro y compromiso) (25).

En Turquía, luego del proceso de adaptación de la escala, se presentaron resultados de un análisis factorial confirmatorio con las respuestas de 250 estudiantes universitarios, y se vieron adecuados índices para ajuste del modelo de cinco dimensiones. El coeficiente de consistencia interna para las dimensiones estuvo entre $\alpha=.62$ y $\alpha=.83$ y para el total de la escala fue $\alpha=.82$ 
(30). En síntesis, los estudios previos reportan adecuados índices de consistencia interna en los distintos grupos de estudiantes universitarios. Con respecto a la estructura del cuestionario, se reportan diversas estructuras factoriales en los estudios analizados (de tres y de cinco factores).

La relevancia en la construcción de medidas basadas en principios psicométricos se observa en el desarrollo de escalas de bienestar multidimensional sobre el uso de procedimientos estadísticos para el análisis de instrumentos de medidas, lo que permite la confirmación de modelos teóricos para describir o explicar los datos empíricos $(17,31)$. Teniendo en cuenta lo anterior, el presente estudio tiene como objetivo estimar las propiedades psicométricas del cuestionario PERMA-Profiler en una muestra estudiantes universitarios chilenos.

\section{Materiales y métodos}

S e realizó una investigación no experimental, de carácter descriptivo de tipo instrumental, con Sla que se pretendió evaluar las propiedades psicométricas de instrumentos de medida $(32,33)$.

\section{Participantes/muestra}

La muestra estuvo conformada por 1462 estudiantes universitarios de una universidad tradicional perteneciente al Consejo de Rectores (сRUCH), ubicada al sur de Chile (657 mujeres; 790 hombres; 15 no respondieron). El promedio de edad fue de 19.09 ( $\mathrm{DE}=1.7$ ). Los estudiantes eran de primer año de las cohortes de ingreso 2017 y 2018 y estaban distribuidos en 48 carreras de distintas áreas. Se realizó un muestreo no probabilístico por cuotas según el área científica de las facultades (tabla 1).

Tabla 1. Distribución de participantes por área científica

\begin{tabular}{lc}
\hline \multicolumn{1}{c}{ Áreas } & Cantidad \\
\hline Ciencias de la educación & 188 \\
Ciencias de la ingeniería & 767 \\
Humanidades, artes y ciencias sociales & 139 \\
Ciencias económicas, administrativas y jurídicas & 43 \\
Ciencias básicas & 160 \\
Ciencias agrícolas, forestales y veterinaria & 165 \\
Total & 1462 \\
\hline
\end{tabular}

\section{Instrumento}

El PERMA-Profiler es una escala que mide los cinco pilares del bienestar propuestos en la teoría PERMA (21): “positive emotions, engagement, relationships, meaning, achievement”, unido a tres 
variables de contraste (las emociones negativas, la soledad y la percepción de salud) (23). Está conformado por 23 reactivos de autorreporte, respondidos con una escala de Likert que van en un rango de 0 (menor puntuación) a 10 (mayor puntuación).

La dimensión de emociones positivas mide las tendencias generales hacia la satisfacción y la alegría. El compromiso se refiere a la presencia de absorción, dedicación y vigor; los niveles muy altos de compromiso se conocen como el estado de "flujo", en el cual la persona está tan absorta en una actividad que pierde todo sentido del tiempo. Las relaciones positivas describen la medida en que la persona recibe y da apoyo a otros, así como la satisfacción y la sensación de sentirse apreciada en las relaciones que establece. El propósito se refiere a los sentimientos dellevar una vida con propósito, sentido y valor; esta dimensión evalúa la sensación de que la vida importa. El logro hace referencia a la frecuencia en que la persona avanza y alcanza sus metas, así como la capacidad de asumir responsabilidades; implica trabajar para alcanzar objetivos y sentirse capaz de completar tareas y responsabilidades diarias. Quince items del PERMA-Profiler permiten el análisis de las dimensiones que conforman la teoría de PERMA.

Aunque no forman parte del modelo PERMA, este instrumento evalúa tres dimensiones de contraste (salud, emociones negativas y soledad). La salud física es considerada otra parte importante del bienestar; esta dimensión mide el sentido subjetivo de sentirse bien y saludable todos los días. Las emociones negativas se refieren a la presencia de ansiedad, tristeza y enojo. Las dimensiones del PERMA, y las dimensiones de emociones negativas y percepción de salud están constituidas por tres ítems cada una. La soledad es evaluada con un ítem, que mide la percepción del sujeto sobre la presencia de esta en su vida (21). Con el PERMA-Profiler se obtienen medidas específicas para cada dimensión y una medida global (18).

Los análisis factoriales confirmatorios en la validación de la escala reportaron valores de Root Mean Square Error of Aproximación (RMSEA) y de Standardized Root Mean Residual (SRMR) o, en español, la Raíz Media Residual Estandarizada dentro de lo estimado teóricamente (RMSEA $=.06, \mathrm{SRMR}=.03, \mathrm{CFI}=.97, \mathrm{TLI}=.96, \chi 2=10.606, \mathrm{df}=80)$. Las cargas factoriales oscilaban desde .36 hasta .93, lo cual confirma los cinco elementos propuestos en el modelo teórico. Con respecto a los índices de fiabilidad en estudiantes universitarios, los valores de consistencia interna se ubicaron desde $\alpha=.73$ hasta $\alpha=.90$ en las dimensiones que componen la escala, y de $\alpha=.92 \mathrm{a}$ $\alpha=.94$ para la escala completa (21).

En el proceso de validación oficial, el PERMA-Profiler presentó correlaciones positivas con escalas de florecimiento y satisfacción con la vida $(r=.78)$ y correlaciones negativas con escalas que miden estrés percibido $(\mathrm{r}=-.55)$, soledad $(\mathrm{r}=-.46)$ y depresión $(\mathrm{r}=-.61)(21)$. Estas características de validez no fueron evaluadas en el presente trabajo.

Para conocer las variables sociodemográficas, se solicitó a los participantes reportar, en el mismo cuadernillo de respuestas, información relativa a sexo, edad, carrera y año de inicio de los estudios universitarios. 


\section{Procedimiento}

Primero, se solicitó a los autores del PERMA-profiler su consentimiento para la validación en el contexto chileno. Se utilizó la versión en español aplicada en el estudio original, utilizada para evaluar a una muestra de estudiantes universitarios mexicanos (21). Por ser este trabajo parte de una investigación más amplia, se modificó la escala de respuesta a siete opciones para que los estudiantes comprendieran más fácilmente el cuadernillo de respuestas. El tiempo de la aplicación no superó los 25 minutos. El instrumento fue aplicado en papel en la sala de clases durante los años académicos 2017 y 2018, luego de la autorización del comité de ética de la universidad, decanos y jefes de cada carrera participante. Además, se les solicitó a los estudiantes la firma de un consentimiento informado antes de responder la escala.

\section{Análisis de los datos}

Se utilizó el programa spss v.20 para la realización de los estadísticos descriptivos, medidas de tendencia central y correlación de Pearson. Para la estimación de la confiabilidad, se emplearon el índice de consistencia interna de alpha de Cronbach y el omega de McDonald; la obtención de este último fue con el programa estadístico JASP V0.8. El análisis de la validez de constructo fue por medio de análisis factorial confirmatorio con el software estadístico MPlus. La estimación de parámetros empleada fue el método de máxima verosimilitud robusto. Para la evaluación del ajuste, se emplearon los índices de chi-cuadrado con el que se esperaba encontrar valores no significativos (debido a la cantidad de participantes, existe cierto consenso en la sensibilidad que tendría este procedimiento estadístico como el tamaño muestral, siendo común obtener significación estadística en muestras grandes $>200$ ). Se consideraron valores menores a .05-.08 en la raíz cuadrada media de error de aproximación (RMSEA) y un intervalo de confianza al $90 \%$ (I.C.) entre .05 y .08; el índice de ajuste comparativo (сғI) y índice de Tucker-Lewis (TLI) considerando obtener valores superiores a $.95(34,35)$.

\section{Resultados}

$A_{\text {ponen la escala perma-Profiler (tabla 2). Se obtuvieron puntuaciones bajas en los ítems }}^{\text {continuación, se presentan los estadísticos descriptivos de los veintitres ítems que com- }}$ pertenecientes a la dimensión de emociones negativas y para el ítem que evalúa la percepción de soledad; esto indica una menor presencia de estos aspectos en la vida de los estudiantes universitarios.

Con respecto a la distribución de las respuestas de los estudiantes, los valores de asimetría y curtosis de cada uno de los ítems en su mayoría fueron negativos y se encontraron en 
rangos aceptables, puesto que valores de asimetría superiores a tres y de curtosis superiores a diez sugieren que los datos deberían transformarse (36).

Tabla 2. Estadísticos descriptivos sobre los ítems del PERMA-profiler

\begin{tabular}{lcccccc}
\cline { 2 - 6 } & Media & $\begin{array}{c}\text { Desv. } \\
\text { Desviación }\end{array}$ & \multicolumn{2}{c}{ Asimetría } & \multicolumn{2}{c}{ Curtosis } \\
\cline { 2 - 6 } & Estadístico & Estadístico & Estadístico & Desv. Error & Estadístico & Desv. Error \\
\hline Emociones positivas 1 & 5.04 & 1.26 & -0.44 & 0.06 & -0.53 & 0.13 \\
Emociones positivas 2 & 4.95 & 1.42 & -0.47 & 0.06 & -0.58 & 0.13 \\
Emociones positivas 3 & 4.92 & 1.28 & -0.42 & 0.06 & -0.37 & 0.13 \\
Compromiso 1 & 4.51 & 1.39 & -0.16 & 0.06 & -0.75 & 0.13 \\
Compromiso 2 & 5.01 & 1.32 & -0.57 & 0.06 & -0.27 & 0.13 \\
Compromiso 3 & 5.86 & 1.25 & -1.15 & 0.06 & 0.75 & 0.13 \\
Relaciones 1 & 4.83 & 1.55 & -0.34 & 0.06 & -0.86 & 0.13 \\
Relaciones 2 & 5.12 & 1.46 & -0.44 & 0.06 & -0.66 & 0.13 \\
Relaciones 3 & 4.95 & 1.47 & -0.48 & 0.06 & -0.58 & 0.13 \\
Propósito 1 & 4.89 & 1.56 & -0.52 & 0.06 & -0.58 & 0.13 \\
Propósito 2 & 4.89 & 1.52 & -0.40 & 0.06 & -0.77 & 0.13 \\
Propósito 3 & 4.92 & 1.47 & -0.50 & 0.06 & -0.48 & 0.13 \\
Logro 1 & 4.79 & 1.40 & -0.32 & 0.06 & -0.63 & 0.13 \\
Logro 2 & 4.57 & 1.35 & -0.20 & 0.06 & -0.74 & 0.13 \\
Logro 3 & 5.01 & 1.30 & -0.43 & 0.06 & -0.49 & 0.13 \\
Emociones negativas 1 & 4.51 & 1.59 & -0.17 & 0.06 & -0.94 & 0.13 \\
Emociones negativas 2 & 3.12 & 1.35 & 0.79 & 0.06 & 0.15 & 0.13 \\
Emociones negativas 3 & 3.24 & 1.36 & 0.79 & 0.06 & -0.05 & 0.13 \\
Salud 1 & 5.25 & 1.26 & -1.05 & 0.06 & 0.94 & 0.13 \\
Salud 2 & 4.52 & 1.53 & -0.45 & 0.06 & -0.55 & 0.13 \\
Salud 3 & 5.10 & 1.39 & -0.87 & 0.06 & 0.23 & 0.13 \\
Soledad único & 3.21 & 1.48 & 0.64 & 0.06 & -0.26 & 0.13 \\
Felicidad único & 5.11 & 1.27 & -0.56 & 0.06 & -0.18 & 0.13 \\
\hline
\end{tabular}

En la tabla 3 se presentan las correlaciones obtenidas entre las dimensiones que constituyen al PERMA-Profiler. Los resultados encontrados permiten afirmar que existen correlaciones positivas, significativas y de adecuada magnitud para las dimensiones que conforman las dimensiones del PERMA. Estos hallazgos son similares a los encontrados en la versión original de la escala (21), lo que muestra adecuado poder discriminante de cada dimensión, puesto que los niveles de correlación se ubicaron entre $\mathrm{r}=.40$ a r=.80 (37). Las correlaciones encontradas confirman no ser lo suficientemente altas para presentar multicolinealidad entre las dimensiones (36).

En el análisis de validez discriminante, las dimensiones del PERMA presentan correlaciones inversas, significativas y de baja magnitud con respecto a la dimensión de emociones negativas, 
lo que indica que a mayores puntuaciones en las dimensiones de emociones positivas, propósito, relaciones y logro, los estudiantes tendrán menor presencia de síntomas de ansiedad, tristeza y enojo, a excepción de la dimensión compromiso (E) que presentó una correlación muy baja y no significativa. En referencia a la validez convergente, se presentan correlaciones positivas, significativas, de baja magnitud con respecto a la percepción de salud. Esto podría indicar que, a mayores niveles en PERMA, mayor será el sentimiento subjetivo de los estudiantes de sentirse saludables (tabla 3).

Desde el punto de vista psicométrico, los valores encontrados permiten solo afirmar adecuados niveles de validez convergente con respecto a la correlación entre la dimensión de emociones positivas y la dimensión de percepción de salud (37).

Tabla 3. Correlaciones de Pearson para las dimensiones del PERMA-Profiler

\begin{tabular}{lcccccc}
\hline & $\begin{array}{c}\text { Emociones } \\
\text { positivas }\end{array}$ & Compromiso & $\begin{array}{c}\text { Relaciones } \\
\text { positivas }\end{array}$ & Propósito & Logro & $\begin{array}{c}\text { Emociones } \\
\text { negativas }\end{array}$ \\
\hline Emociones positivas & & & & & & \\
Compromiso & $.477^{* * *}$ & & & & \\
Relaciones positivas & $.646^{* * *}$ & $.408^{* *}$ & & & \\
Propósito & $.717^{* * *}$ & $.502^{* *}$ & $.544^{* *}$ & & & \\
Logro & $.653^{* * *}$ & $.478^{* *}$ & $.508^{* *}$ & $.767^{* *}$ & & \\
Emociones negativas & $-.403^{* *}$ & -0.018 & $-.241^{* *}$ & $-.227^{* *}$ & $-.190^{* *}$ & \\
Percepción de salud & $.361^{* * *}$ & $.173^{* *}$ & $.259^{* *}$ & $.330^{* *}$ & $.329^{* *}$ & $-.270^{* *}$ \\
\hline
\end{tabular}

Nota: ${ }^{* *}$ La correlación es significativa en el nivel 0.01 (bilateral).

El presente estudio buscó confirmar la estructura teórica original del PERMA (23) en una muestra de estudiantes universitarios chilenos. Para ello, se analizaron los quince ítems del PERMA-profiler que constituyen las cinco dimensiones principales del cuestionario; los resultados indican la existencia de ajuste en las cinco dimensiones teóricas propuestas en el estudio original (tabla 4).

Tabla 4. Estimaciones del PERMA-Profiler

\begin{tabular}{cccccccc}
\hline & $\mathbf{X}^{2}$ & D $\boldsymbol{N}$ & RMSEA & (90\% CI) & TLI & CFI \\
\hline Modelo & $411.953^{* *}$ & 80 & .054 & $.049-.059$ & .95 & .94 \\
\hline
\end{tabular}

Nota: N=1462; d f = grados de libertad del modelo; RMSEA = Error Medio Cuadrático de Aproximación; (90\% cI) = Intervalo de confianza del $90 \%$ para RMSEA; TLI= índice de Tucker-Lewis; CFI= Índice de Ajuste Comparativo; ${ }^{* *}$ p $<.01$

Los resultados indicaron un adecuado ajuste del modelo. Se presenta un valor de chi-cuadrado significativo (esperable en muestras grandes), el RMSEA se encuentra dentro del 
rango recomendado (.05-.08), al igual que los índices de ajuste alternativo de Tucker-Lewis y ajuste comparativo $(.90-.95)(34,35)$.

Los cinco factores propuestos para el análisis presentaron cargas significativas para cada dominio, lo cual confirma las dimensiones de (a) emociones positivas, (b) compromiso, (c) relaciones positivas, (d) propósito y (e) logro. Se presentan altas cargas para la mayoría de los elementos que integran el cuestionario, a excepción del ítem C1: “¿Con qué frecuencia te sientes absorbido(a) en lo que estás haciendo?”, que pertenece a la dimensión de compromiso (figura 1).

Figura 1. Diagrama de estructura del PERMA-Profiler

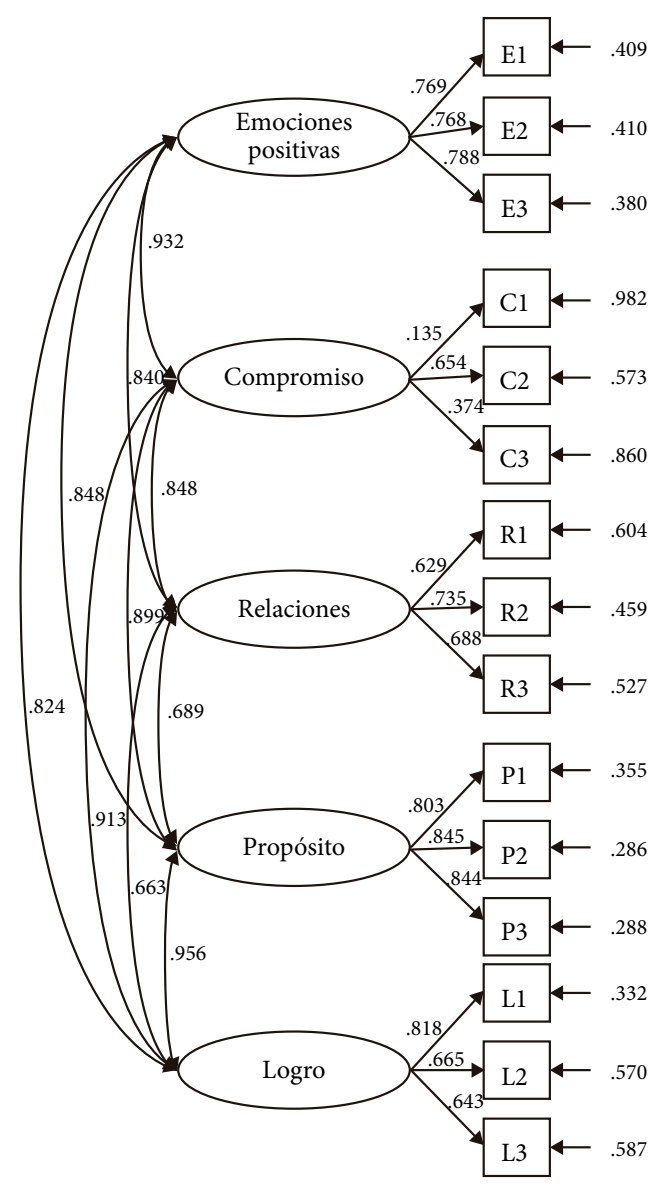

En la tabla 5 se presentan los resultados de la estimación de la confiabilidad de las respuestas de los estudiantes participantes. Se empleó el coeficiente de fiabilidad evaluado por medio del índice de consistencia interna alfa ( $\alpha$ ) de Cronbach y el Omega ( $\omega)$ de McDonald, en los cuales teóricamente se espera encontrar puntuaciones superiores a .70 para cada una de las dimensiones y para el total de la escala (37). Los resultados que se encontraron en el presente estudio están dentro del parámetro esperado para la mayoría de las dimensiones 
de la escala y la puntuación total. La dimensión de compromiso presentó un índice de consistencia interna bajo ( $\alpha=.36$ ); estos resultados coinciden con el bajo peso del ítem "¿Con qué frecuencia te sientes absorbido(a) en lo que estás haciendo?”(C1) que pertenece a la misma dimensión, lo que podría explicar este resultado.

Tabla 5. Coeficientes de confiabilidad de las dimensiones y el total del PERMA-Profiler

\begin{tabular}{lcc}
\hline \multicolumn{1}{c}{ Dimensiones } & Alpha de Cronbach & $\begin{array}{c}\text { Omega } \\
\text { Mc Donald's }\end{array}$ \\
\hline Emociones positivas & .824 & .827 \\
Compromiso & .360 & .379 \\
Relaciones positivas & .732 & .746 \\
Propósito & .874 & .875 \\
Logros & .757 & .759 \\
Salud & .846 & .854 \\
Emociones negativas & .652 & .663 \\
PERMA Total & .918 & .923 \\
\hline
\end{tabular}

\section{Discusión}

$\mathrm{E}$ l objetivo del presente trabajo fue estimar las propiedades psicométricas del cuestionario PERMA-Profiler en una muestra de estudiantes universitarios chilenos. Las puntuaciones obtenidas por los estudiantes universitarios son similares a las de otros estudios realizados en el contexto latinoamericano, aunque se presenta mayor presencia de emociones positivas que emociones negativas en estudiantes $(3,38)$. También se sugiere que altos niveles de bienestar en grupos de personas latinoamericanas están presentes cuando existen mayores niveles de instrucción (39).

Se vieron adecuadas correlaciones entre las dimensiones del PERMA-Profiler y la puntuación total de la escala, se presentaron resultados cercanos a los encontrados en la versión original y similares a los encontrados en la validación de participantes de Malasia $(21,25)$. Estos hallazgos permiten confirmar que las emociones positivas, el compromiso, las relaciones, el propósito y la percepción de logro son constructos relacionados que representan una visión multidimensional del bienestar.

Con respecto a la validez convergente, al analizar los niveles de correlación del PERMA-Profiler con las variables de contraste de percepción de salud y emociones negativas, se encontró que los resultados reportados por los estudiantes universitarios chilenos tenían correlaciones de menor magnitud a los encontrados en la versión original (emociones negativas $r=-.34$ 
hasta $\mathrm{r}=-.52$ y percepción de salud $\mathrm{r}=.31$ hasta $\mathrm{r}=.43$ ), aunque se conservó la dirección y significación en estas relaciones. Se mantuvo un adecuado nivel de correlación con respecto a la dimensión de emociones positivas con la dimensión de percepción de salud (21).

Considerando que la versión original de la escala presenta un ajuste adecuado en el modelo de cinco factores y siguiendo las recomendaciones de Pérez-Gil, quien argumenta que la realización de análisis factorial exploratorio y análisis factorial confirmatorio en conjunto no añade una mejor información sobre la validez de constructo de la estructura factorial, y sabiendo pues, que el análisis factorial confirmatorio busca corroborar un modelo teóricamente plausible para describir o explicar los datos empíricos, en el presente estudio se realizó un análisis factorial confirmatorio con los quince ítems que conforman las cinco dimensiones del PERMA-Profiler (21, 31). Estas presentaron un adecuado ajuste y mostraron valores dentro de los rangos de puntuaciones aceptados en la literatura psicométrica y estadística (34, 35, 40). Estos resultados confirman la estructura original con la misma cantidad de ítems y dimensiones y en otras versiones analizadas con estudiantes universitarios $(21,30)$.

El ítem C1, “¿Con qué frecuencia te sientes absorbido(a) en lo que estás haciendo?”, presentó una carga factorial menor (r.30), a lo esperado teóricamente (37). Sin embargo, se decidió mantener este ítem en el análisis por mantener significación estadística de la estructura del cuestionario. Posiblemente este resultado se deba a que los estudiantes universitarios no comprendieron las preguntas de esta dimensión de la forma que se espera teóricamente y que existan diferencias culturales con respecto a esta pregunta. Desde la teoría del engagement, el término de absorción puede ser visto de dos maneras: primero de forma positiva, en la que la persona puede estar intrínsecamente motivada, y segundo de forma perjudicial, en la que la absorción es una forma de adicción al trabajo (41). En ese caso sería provechoso el desarrollo de entrevistas adicionales que permitan evaluar la comprensión y significado que los estudiantes les atribuyen a las preguntas de esta dimensión.

La consistencia interna para la escala total fue favorable $(\alpha=.91 ; \omega=.92)$, pues se presentaron niveles adecuados según lo esperado teóricamente y coincidente con lo reportado en la versión original ( $\alpha=.92)(21,37,40,41)$. Además, se presentaron mayores puntuaciones de consistencia interna que los presentados en la versión adaptada para estudiantes universitarios de Turquía ( $\alpha=.82$ ) (30). Con respecto a los niveles de consistencia reportados en las dimensiones del instrumento de medida, los resultados fueron adecuados teóricamente para las dimensiones de emociones positivas ( $\alpha=.82$ ), relaciones $(\alpha=.73$ ), propósito ( $\alpha=.87)$ y logro ( $\alpha=.75)$, percepción de salud $(a=.84)$ y emociones negativas $(\alpha=.65)$. Solo la dimensión de compromiso no presentó adecuados niveles de consistencia interna en esta muestra de participantes ( $\alpha=.36$ ), esto debido al efecto de variabilidad en la comprensión de los ítems que componen esta dimensión. En general, la dimensión de compromiso fue la que presentó menores puntuaciones con respecto al resto de las dimensiones de la escala; este resultado también fue presentado en otros estudios $(21,30)$. 
Investigaciones futuras deben examinar la presentación y redacción de los ítems de la dimensión de compromiso para ampliar la comprensión de esta dimensión en el instrumento. Además de la evaluación más profunda de la validez predictiva y concurrente de esta escala con otros instrumentos utilizados en el contexto educativo chileno (satisfacción con la vida, afectos positivos, entre otros), así como la estabilidad de las respuestas en el tiempo, con el propósito de continuar el análisis psicométrico de la escala es importante dejar claro que este proceso de adaptación fue realizado en un solo nivel educativo (universitarios). En tal sentido, es recomendable realizar investigaciones de validación de este instrumento en participantes con características distintas a las empleadas en el presente estudio con la finalidad de ampliar la aplicabilidad de esta escala en distintos escenarios.

Los resultados de este estudio son de importancia para la promoción de la salud mental en el contexto de la educación superior. Los consejeros educativos y departamentos de salud estudiantil, así como otros departamentos, podrían utilizar el PERMA-Profiler para la medición de salud de los estudiantes como una herramienta para informar y apoyarlos en el conocimiento de su bienestar y salud mental. Además, se podría utilizar para evaluar su relación con otras variables presentes en el contexto universitario como rendimiento académico, autoeficacia, vitalidad, optimismo, ansiedad y como una herramienta válida para el diseño de intervenciones de salud en este contexto.

En conclusión, el PERMA-Profiler posee adecuadas propiedades psicométricas para su uso en estudiantes universitarios en Chile. Se identificaron presencia de mayores puntuaciones de emociones positivas sobre emociones negativas en las respuestas de los estudiantes participantes. En general, se presentaron adecuados niveles de bienestar. Se identificaron correlaciones positivas entre las dimensiones que lo constituyen y la percepción de salud. Las dimensiones del PERMA-profiler presentan correlaciones inversas con las emociones negativas, tal y como se espera teóricamente. En términos de estructura, se confirma la propuesta de cinco factores evaluadas en la versión original del instrumento. Se identificaron adecuados índices de consistencia interna en la mayoría de las dimensiones y en el total de la escala.

\section{Contribución de los autores}

T os autores contribuyeron en la concepción y el diseño de la investigación, así como en la ـadquisición, análisis e interpretación de los datos. Además, en la planeación del artículo o revisión de contenido intelectual importante y aprobación final de la versión por ser publicada. 


\section{Conflicto de intereses}

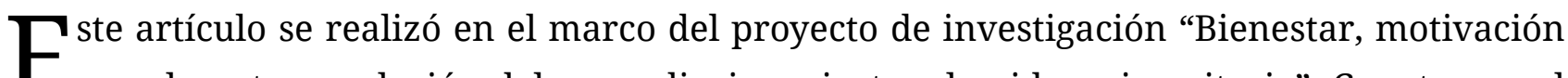
Epara la autorregulación del aprendizaje y ajuste a la vida universitaria”. Cuenta con el financiamiento de Becas Doctorado Nacional Conicyt Folio 21180295 y con apoyo del proyecto Fondecyt 1161502, modelo explicativo de la permanencia y el abandono de los estudios universitarios, basado en procesos cognitivo motivacionales.

\section{Referencias}

1. Colleen SC, Kirsch AC, Dickson DA, Bryant FB. Negotiating the transition to college. Emerg Adulthood. 2014;2(3):195-210. Doi: 10.1177/2167696814521808

2. Stallman HM. Psychological distress in university students: A comparison with general population data. Aust Psychol. 2010;45(4):249-257. Doi: 10.1080/00050067.2010.482109

3. Barrantes-Brais K, Ureña-Bonilla P. Bienestar psicológico y bienestar subjetivo en estudiantes universitarios costarricenses. Revista Intercontinental de Psicología y Educación. 2015;17(1):101-123.

4. Moreta R, Gabior I, Barrera L. El bienestar psicológico y la satisfacción con la vida como predictores del bienestar social en una muestra de universitarios ecuatorianos. Salud \& Sociedad. 2017;8(2):172-184. Doi: 10.22199/S07187475.2017.0002.00005

5. Matalinares ML, Díaz G, Arenas C, Raymundo O, Baca D, Uceda J, Yaringaño J. Afrontamiento del estrés y bienestar psicológico en estudiantes universitarios de Lima y Huancayo. Revista de Investigación en Psicología. 2016; 19(2):123-143. Doi: 10.15381/ rinvp.v19i2.12894

6. Schotanus-Dijkstra M, Ten Have M, Lamers SMA, de Graaf R, Bohlmeijer ET. The longitudinal relationship between flourishing mental health and incident mood, anxiety and substance use disorders. Eur J Public Health. 2016;27(3):563-568. Doi: 10.1093/eurpub/ ckw202

7. Mega C, Ronconi L, De Beni R. What makes a good student? How emotions, self-regulated learning, and motivation contribute to academic achievement. J Educ Psychol 2014;106(1):121-131. Doi: http://dx.doi.org/10.1037/a0033546

8. González-Brignardello MP, Elvira-Paniagua AS. ¿Puede amortiguar el Engagement los efectos nocivos de la procrastinación académica? Acción Psicológica. 2013;10(1):115134. Doi: 10.5944/ap.10.1.7039

9. Cazan AM. Self regulated learning strategies - predictors of academic adjustment. Procedia Soc Behav Sci. 2012;33:104-108. Doi: 10.1016/j.sbspro.2012.01.092

10. Nightingale SR, Roberts S, Tariq V, Appleby Y, Barnes L, Harris RA, Dacre-Pool L, Qualter P. Trajectories of university adjustment in the United Kingdom: Emotion management and emotional self-efficacy protect against initial poor adjustment. Learn Individ Differ. 2013;27:174-181. Doi: 10.1016/j.lindif.2013.08.004 
11. Oriol-Granado X, Mendoza-Liria M, Covarrubias CG, Molina-López VM. Emociones positivas, apoyo a la autonomía y rendimiento de estudiantes universitarios: el papel mediador del compromiso académico y la autoeficacia. Revista de Psicodidáctica. 2016; 22(1):45-53. Doi: 10.1387/RevPsicodidact.14280

12. Coffey JK, Wray-Lake L, Mashek D, Branand B. A multi-study examination of well-being theory in college and community samples. J Happiness Stud. 2016;17(1):187-211. Doi: 10.1007/s10902-014-9590-8

13. Gray J, Hackling M. Wellbeing and retention: A senior secondary student perspective. Aust Educ Res. 2009;36(2):119-145. Doi: 10.1007/BF03216902

14. Jongbloed J, Andres L. Elucidating the constructs happiness and wellbeing: A mixed-methods approach. International Journal of Wellbeing. 2015;5(3):1-20. Doi: 10.5502/ijw. v5i3.1

15. Keyes CL, Shmotkin D, Ryff CD. Optimizing well-being: the empirical encounter of two traditions. Journal of Personality and Social Psychology. 2002;82(6):1007-1022. Doi: 10.1037//0022-3514.82.6.1007

16. Kern ML, Waters LE, Adler A, White MA. A multidimensional approach to measuring well-being in students: Application of the PERMA framework. J Posit Psychol. 2015;10(3):262271. Doi: $10.1080 / 17439760.2014 .936962$

17. Huppert FA. The state of wellbeing science. Concepts, measures, interventions, and policies: U.K. John Wiley \& Sons; 2014.

18. Hone LC, Jarden A, Schofield GM, Duncan S. Measuring flourishing: The impact of operational definitions on the prevalence of high levels of wellbeing. International Journal of Wellbeing. 2014;4(1):62-90. Doi: 10.5502/ijw.v4i1.4

19. Adler A, Seligman ME. Using wellbeing for public policy: Theory, measurement, and recommendations. International Journal of Wellbeing. 2016;6(1):1-35. Doi: 10.5502/ijw. v6i1.429

20. Durand M. The oEcd Better life initiative: How's life? and the measurement of well-being. Review of Income and Wealth. 2015;61(1):4-17. Doi: 10.1111/roiw.12156

21. Butler J, Kern ML. The PERMA-Profiler: A brief multidimensional measure of flourishing. International Journal of Wellbeing. 2016;6(3):1-48. Doi: 10.5502/ijw.v6i3.526

22. Cobo-Rendón R, Pérez Villalobos MV, Hernández H, Aslan-Parra J. Modelos multidimensionales del bienestar en contextos de enseñanza-aprendizaje: una revisión sistemática. Ecos de la Academia 2017;6(1): 13-28.

23. Seligman M. La vida que florece. Madrid Ediciones B; 2011.

24. Jayawickreme E, Forgeard MJC, Seligman MEP. The Engine of Well-Being. Rev Gen Psychol. 2012;16(4):327-42. Doi: 10.1037/a0027990

25. Khaw D, Kern ML. A cross-cultural comparison of the PERMA model of well-being. Undergraduate Journal of Psychology at Berkeley, University of California. 2014;8:1023. Disponible en: http://ujpb.org/vol8/edition8.pdf\#page $=22$

26. Kun Á, Péter B, Gerakné Krasz K. Development of the work-related well-being questionnaire based on Seligman's PERMA model. Periodica Polytechnica Social and Management Sciences. 2017;25(1):56-63. Doi: 10.3311/PPso.9326 
27. Kern ML, Benson L, Steinberg EA, Steinberg L. The ЕРосн measure of adolescent well-being. Psychological Assessment. 2016;28(5):586-97. Doi: 10.1037/pas0000201

28. Tansey T, Smedema S, Umucu E, Iwanaga K, Jia-Rung W, Da Silva-Cardoso E, Strauser D. Assessing college life adjustment of students with disabilities: Application of the PERMA Framework. Rehabil Couns Bull. 2018;61(3):131-42. Doi: 10.1177/0034355217702136

29. Umucu E, Jia-Rung W, Sanchez J, Brooks JM, Chiu C-Y, Tu W-M, Chan F. Psychometric validation of the PERMA-profiler as a well-being measure for student veterans. J Am Coll Health. 2019:1-7. Doi: 10.1080/07448481.2018.1546182

30. Ayse EB. Adaptation of the PERMA well-being Scale into Turkish: Validity and reliability studies. Educational Research and Reviews. 2018;13(4):129-135. Doi: 10.5897/ERR2017.3435

31. Pérez-Gil JA, Chacón-Moscoso S, Moreno-Rodríguez R. Validez de constructo: el uso de análisis factorial exploratorio-confirmatorio para obtener evidencias de validez. Psicothema. 2000;12(2):442-446.

32. Ato M, López-Gárcia J, Benavente A. Un sistema de clasificación de los diseños de investigación en psicología. Anales de Psicología. 2013;29(3):1038-1059. Doi: 10.6018/ analesps.29.3.178511

33. Montero I, León OG. Sistema de clasificación del método en los informes de investigación en Psicología. Int. J Clin Health Psychol 2005;5(1):115-127.

34. Hair JF, Anderson RE, Tatham RL, Black WC. Análisis multivariante. $5^{\circ}$ ed. Madrid: Prentice Hall; 1999.

35. Brown TA. Confirmatory factor analysis for applied research. $2^{\circ}$ ed. Nueva York: Guilford Publications; 2014.

36. Kline RB. Principles and practice of structural equation modeling. $4^{\circ}$ ed. Nueva York: Guilford publications; 2015.

37. Luján-Tangarife J, Cardona-Arias J. Construcción y validación de escalas de medición en salud: revisión de propiedades psicométricas. Archivos de Medicina. 2015;11(3):110. Doi: $10.3823 / 1251$

38. Pastrana MP, Salazar-Piñeros FA. Perfil PERMA en una muesta de jóvenes voluntarios colombianos. Revista Katharsis. 2016;(22):13-34.

39. Aguilar-Sizer M, Lima-Castro S, Peña-Contreras E, Cedillo-Quizphe C, Bueno-Pacheco A. Variables sociodemográficas relacionadas con el bienestar en personas con o sin discapacidad. Revista cientifica Maskana 2017;8:37-47.

40. Streiner DL, Norman GR, Cairney J. Health measurement scales: a practical guide to their development and use. $5^{\circ}$ ed. Oxford: Oxford University Press; 2015.

41. Juárez García A. Engagement laboral, una concepción científica: entrevista con Wilmar Schaufeli. Liberabit. 2015;21(2):187-94.

42. Ramada-Rodilla JM, Serra-Pujadas C, Delclós-Clanchet GL. Adaptación cultural y validación de cuestionarios de salud: revisión y recomendaciones metodológicas. Salud pública de México. 2013;55(1):57-66. 